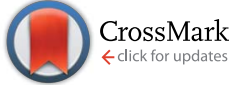

Cite this: RSC Adv., 2017, 7, 2594

Received 19th October 2016 Accepted 20th December 2016 DOI: $10.1039 / c 6 r a 25448 a$ www.rsc.org/advances

\section{Mori Cortex regulates P-glycoprotein in Caco-2 cells and colons from rats with experimental colitis via direct and gut microbiota-mediated mechanisms $\dagger$}

\begin{abstract}
Wanghui Jing, ${ }^{\text {ab }}$ Xuejiao Gao, ${ }^{a}$ Beilei Han, ${ }^{a}$ Bin Wei, ${ }^{a}$ Nan Hu, ${ }^{a}$ Sai Li, ${ }^{a}$ Ru Yan ${ }^{\star a c}$ and Yitao Wang ${ }^{\text {ac }}$

P-Glycoprotein dysregulation and microbial imbalance have been implicated in inflammatory bowel diseases. Here we found that oral dosing of Mori Cortex, the root bark of Morus alba L. markedly alleviated inflammatory responses, reinstated microbial balance, and enhanced P-glycoprotein (P-gp) expression in rat colitis (UC) induced by oral administration of dextran sulfate sodium. The effects of Mori Cortex extract (MCE) on colon P-gp were examined using Caco-2 cells which revealed a timedependent regulatory profile. The distinct effects on P-gp by individual main components may account for the direct biphasic effects of MCE. The involvement of gut microbiota in P-gp regulation by MCE was assessed by incubating the culture supernatant (CS) of fecal bacteria from normal, UC or MCE pretreated rats with Caco-2 cells. Interestingly, compared to normal CS, UC CS but not MCE CS diminished P-gp expression in Caco-2 cells, and this down-regulation could be reversed by pretreatment of Caco- 2 cells with MCE. Moreover, MCE CS treated Caco-2 cells generated proinflammatory IL-1 $1 \beta$ and IL- 8 comparable to that of the normal group and lower than the UC group, whereas, the anti-inflammatory IL-10 stimulated by MCE CS was significantly higher than the UC CS. In conclusion, MCE alleviated colitis-like symptoms and enhanced the intestinal epithelial integrity ( $\mathrm{P}$-gp up-regulation) in experimental colitis. The mechanisms involve both a direct effect and a gut microbiota-mediated pathway.
\end{abstract}

\section{Introduction}

Inflammatory bowel disease (IBD) is a chronic inflammatory disorder which affects the gastrointestinal tract with an everincreasing incidence and tendency to more severe clinical phenotypes. IBD is classified into two primary types that show distinct pathologic features, namely ulcerative colitis (UC) and Crohn's disease (CD). The etiology of IBD is still poorly understood. Various environmental and host factors, such as genetic, epithelial, immune and nonimmune factors, are believed to be involved. Recently, impaired intestinal epithelial integrity of the intestinal epithelium is regarded as one of the major factors of IBD pathogenesis. ${ }^{1,2}$ Both UC and CD were presumed to be a consequence of a defective mucosal barrier and a dysregulated

${ }^{a}$ State Key Laboratory of Quality Research in Chinese Medicine, Institute of Chinese Medical Sciences, University of Macau, Taipa, Macao SAR, China. E-mail: ruyan@ umac.mo

${ }^{b}$ School of Pharmacy, Health Science Center, Xi'an Jiaotong University, No. 76, Yanta West Road, Xi'an, Shannxi, The people's Republic of China

${ }^{c} U M$ Zhuhai Research Institute, No. 1 Software Road, Zhuhai Hi-tech Zone, Guangdong, China

$\dagger$ Electronic supplementary information (ESI) available. See DOI: 10.1039/c6ra25448a immune response to the host's gut microbiota in genetically susceptible individuals. ${ }^{3}$

P-Glycoprotein (P-gp) is an ATP-dependent efflux transporter pump highly expressed in apical surfaces of the intestinal epithelial cells. It constitutes an important component of the intestinal barrier and mediates the efflux of numerous bacterial toxins, carcinogens and drugs from the intestinal mucosa to gut lumen. ${ }^{4}$ P-gp may also have a role in the transport of cytokines, in particular IL-1 $\beta$, IL-2, IL-4 and IFN- $\gamma$, out of activated normal lymphocytes into the surrounding medium, which may regulate a range of physiological responses. ${ }^{5}$ The levels of P-gp expression gradually rise from the duodenum to the distal parts of the intestine with the highest levels of expression in the distal small bowel and colon. ${ }^{6}$ Alteration of P-gp expression and function was reported to be associated with the pathogenesis of several diseases including IBD. Diminished P-gp expression was observed in the gut mucosa of patients with active UC, ${ }^{7}$ while an significantly elevated P-gp expression was found in children with $\mathrm{CD}{ }^{8}$ Furthermore, MDR1 polymorphisms are associated with both CD and UC with a stronger association with UC. ${ }^{9}$ Transgenic mouse with $m d r 1 a$ gene deficiency developed spontaneous colitis resembling human $\mathrm{UC},{ }^{\mathbf{1 0}}$ while those lacking MRP2 and BCRP couldn't. ${ }^{11}$ These findings imply the 
association of P-gp with IBD and make $M D R 1$ a new candidate gene for therapy of IBD.

Gut microbiota harboring in gastrointestinal tract plays an essential role in regulating host immune homeostasis. Shifts in microbial composition lead to immune dysregulation, which is believed to be a critical factor in IBD initiation and progression. ${ }^{12}$ In $\operatorname{mdr} 1 a(-/-)$ mice, the development of an alternate microbial population precedes the development of $\mathrm{IBD},{ }^{13}$ and oral antibiotic treatment could attenuate or reverse colitis induced by mdr1a gene deficiency, ${ }^{14}$ underlining the link between P-gp and gut microbe in pathogenesis of IBD. Certain probiotics, such as Lactobacilli acidophilus and the soluble factors it secreted could stimulate P-gp expression and activity in Caco- 2 cells and in mice with dextran sulfate sodium (DSS)induced colitis. L. acidophilus treatment alleviated the inflammation and prevented the reduction of P-gp expression in the distal colon. ${ }^{15}$ Both antibacterial drugs rifampicin and ciprofloxacine and P-gp inducer spironolactone ameliorated the clinical hallmarks of colitis in 2,4,6-trinitrobenzenesulfonic acid (TNBS)-induced colitis of the rats. ${ }^{\mathbf{1 6}}$ Again, these results shed a light on the potential of P-gp as a new therapeutic target and the involvement of gut bacteria in intestinal P-gp regulation.

Mori Cortex, the root bark of Morus alba L., is traditionally used in China for relieving pulmonary disorders, such as chronic obstructive pulmonary disease, asthma, cough, or as a diuretic agent. It is also a main constitute herb in several compound formulas used for alleviation of GI disorders. ${ }^{17}$ Recent in vivo and in vitro studies revealed a variety of health-promoting effects of this herb and its main components, including anti-inflammatory, anti-oxidant, and anti-microbial activities. ${ }^{18-20}$ The ethanolic extract of $M$. alba L. leave was reported to exhibit significant antiulcerogenic activity in experimentally induced gastric ulcer in rats. ${ }^{21}$ Co-administration of mulberry, the fruits of $M$. alba L., significantly upregulated the P-gp activity in normal rats and consequently decreased the systemic exposure of cyclosporine, a probe substrate of P-gp. ${ }^{22}$ Several components found in Mori Cortex also showed regulatory effects on P-gp expression and activity, ${ }^{23,24}$ while most of the studies were carried out in vitro and some of the results were controversial. The questions that whether Mori Cortex could enhance the P-gp expression and alleviate IBD remain to be addressed.

Therefore, in the present study, the effect of Mori Cortex on colitis was first assessed on a rat model with DSS-induced experimental colitis. The P-gp alteration in colons from rats with colitis as well as in Caco- 2 cells, a human epithelial colorectal adenocarcinoma cell line that naturally expresses the MDR1 gene, in response to Mori Cortex intervention was characterized. The involvement of gut microbiota in the P-gp regulatory effects of Mori Cortex was further delineated in Caco-2 cell model.

\section{Materials and methods}

\subsection{Chemicals and reagents}

Dextran sulfate sodium (DSS; MW 36 000-50 000) was purchased from MP Biomedicals (Solon, OH). Pierce ${ }^{\mathrm{TM}}$ Bicinchoninic Acid
(BCA) protein assay kit was purchased from Thermo Fisher Scientific (Waltham, MA, USA). Anti-P-gp mouse monoclonal antibody (mAb) was purchased from Calbiochem (Darmstadt, Germany). Anti-glyceraldehyde-3-phosphate dehydrogenase (GAPDH) rabbit antibody, goat anti-rabbit IgG horseradish peroxidase (HRP)-conjugated antibody, anti-mouse IgG-HRP conjugated antibody and complete protease inhibitor cocktail were purchased from Santa Cruz Biotechnology, Inc. (Santa Cruz, CA). ECL advanced western blotting detection kit was purchased from GE Healthcare (Becton-Dickinson, NJ). The enzyme-linked immunosorbent assay (ELISA) kits for TNF- $\alpha$, IL-1 $\beta$, IL-4, IL-6, IL-8 and IL-10 were obtained from R\&D Systems (Minneapolis, MN). 4-Nitrophenyl- $\beta$-D-glucopyranoside (PNP-GN), 4-nitrophenyl- $\beta$-D-glucuronide (PNP-GS) and 4-nitrophenol were purchased from Sigma-Aldrich (St. Louis, MO). 3-(4,5Dimethylthiazole-2-ly)-2,5-diphenyl tetrazolium bromide (MTT) was purchased from USB Co. (Cleveland, OH). Rhodamine 123 (Rho123) was purchased from Sigma-Aldrich Co. (St Louis, MO). Dulbecco's Modified Eagle's medium (DMEM), fetal bovine serum (FBS), penicillin-streptomycin and nonessential amino acids were obtained from Gibco BRL Life \& Technologies (Grand Island, NY). All other chemicals used were of analytical grade and obtained commercially. Transwell ${ }^{\circledR}$ plates (12-well, $0.4 \mu \mathrm{m}$ pore size, $1.12 \mathrm{~cm}^{2}$, polycarbonate membrane) were purchased from Corning Costar Co. (Cambridge, MA). Caco-2 cells at passage 19 were obtained from the American Type Culture Collection (ATCC, Rockville, MD).

\subsection{Preparation of Mori Cortex extract}

The preparation of the Mori Cortex extract (MCE) and the chemical profile of the extract obtained using LC-MS/MS analysis have been described in our recent report. ${ }^{25} \mathrm{~A}$ portion of the MCE $(20.0 \mathrm{~g})$ was dissolved in $100 \mathrm{~mL}$ of $50 \%$ propylene glycol solution for the experiment. Prenylflavonoids (mainly morusin, cyclomorusin, sanggenon B, sanggenon D and kuwanon C) and Diels-Alder type adducts (kuwanon G and kuwanon O) present as the main constituents, while stilbenes, 2-arylbenzofuran derivatives and other types, including mulberroside $\mathrm{A}$, mulberroside $\mathrm{C}$, moracin $\mathrm{M}$, moracin $\mathrm{O}$ and moracin $\mathrm{P}$ and morin were the minor components in the extract.

\subsection{Animals}

Male Sprague-Dawley (200-250 g) rats were purchased from the Laboratory Animal Research Center of Chinese University of Hong Kong (Hongkong, China). Eighteen animals were randomly divided into three groups (6 animals in each group) and allocated to individual metabolic cages (Tecniplast, Italy). Animals were housed in an air-conditioned room $\left(23 \pm 2{ }^{\circ} \mathrm{C}\right)$ with a relative humidity of $55 \pm 5 \%$ under a 12/12 hour light/ dark cycle and fed corn and soybean diet. Animals were allowed to get acclimated to the environment for one week prior to experiment. The animal experiments were conducted according to a protocol (file no.: ICMS-AEC-2013-05) approved by the Animal Ethics Committee, the Institute of Chinese Medical Sciences, the University of Macau. 


\subsection{In vivo study}

2.4.1 DSS-induced experimental colitis in rats. In the in vivo study, rats received either drinking water alone (normal group), 1 week (day 0-day 7) DSS stimulation (UC group) or 1 week DSS insult plus 3 week MCE intervention (MCE group). DSS solution at $5 \%(\mathrm{w} / \mathrm{v})$ was freshly prepared daily by dissolving DSS into sterilized distilled drinking water. Rats in MCE group received the MCE 50\% propylene glycol solution $(0.5 \mathrm{~g} / 2.5$ $\mathrm{mL} \mathrm{kg}{ }^{-1}$ ) once daily via oral gavage 1 week prior to initiating DSS stimulation and continued for 3 weeks (day 7-day 14). Meanwhile, animals from the normal and UC groups received the same volume of $50 \%$ propylene glycol solution via oral gavage for 3 weeks.

2.4.2 Daily observation and sample collection. Body weight, stool consistency and rectal bleeding were recorded daily throughout the experimental period according to the disease activity index (DAI) scoring system adopted in our laboratory. ${ }^{26}$ Feces and blood samples were collected from each rat every other day throughout the experiment (day 7-day 14). Blood samples (200 $\mu \mathrm{L}$ each) were obtained from orbital sinus, left at $37^{\circ} \mathrm{C}$ for $30 \mathrm{~min}$, then centrifuged at $1000 \times g$ for $10 \mathrm{~min}$ to obtain serum. On the last day (day 14), animals were sacrificed by decapitation. The colon was removed to exam ulcers in colon lining and measure the length and the weight of colons. One-centimeter of the distal colon was obtained for histological assessment. The rest of colon tissue was cut into pieces and snap frozen by liquid nitrogen for western blot analysis as described below. The spleen was rinsed with icecold normal saline and wiped dry before measurement of the weight.

2.4.3 Histological assessment of colon tissue. Colon tissues were immediately fixed with paraformaldehyde (4\% in PBS) overnight. The fixed tissues were then embedded in paraffin wax, sectioned, and stained with hematoxylin and eosin (H\&E). The histological specimen was examined in $\times 40$ and $\times 400$ fields under a light microscope.

2.4.4 Measurement of myeloperoxidase activity in colon tissue. The myeloperoxidase (MPO) activity in the colon tissue was determined as described in our previous report. ${ }^{26}$ One unit of MPO activity was defined as the amount of enzyme required to convert $1 \mathrm{nmol}$ of hydrogen peroxide to water per minute at room temperature.

2.4.5 Determination of serum cytokines. The concentrations of TNF- $\alpha$, IL-1 $\beta$, IL- 6 , IL-4 and IL-10 in the serum were determined with an ELISA kit from R\&D Systems (Minneapolis, $\mathrm{MN}$ ) following the manufacture's guidance.

2.4.6 16S rRNA-based gut microbiota analysis. Gut bacteria compositions were analyzed using 16S rRNA-based RT-PCR method with minor modifications ${ }^{27,28}$ and amplification sizes were given in Table 1. Briefly, the target bacteria include Bifidobacterium, Enterobacteriaceae, Bacteroides fragilis, Clostridium coccoides, Clostridium leptum. Respective primers ${ }^{29,30}$ and amplification sizes were provided in ESI Table S1. $\dagger$ Fecal DNA was extracted and target genes were absolutely quantified using the ABI 7500 real time PCR instrument (Applied Biosystems, USA). The experiment was performed in triplicate.
Table 1 16S rRNA gene-targeted primers

\begin{tabular}{|c|c|c|c|}
\hline Target bacteria & Primer & Sequence $\left(5^{\prime}-3^{\prime}\right)$ & bp \\
\hline \multirow[t]{2}{*}{ Bifidobacterium } & g-Bifid-F & CTCCTGGAAACGGGTGG & 556 \\
\hline & g-Bifid-R & GGTGTTCTTCCCGATATCTACA & \\
\hline \multirow[t]{2}{*}{ Enterobacteriaceae } & Eco1457F & CATTGACGTTACCCGCAGAAGAAGC & 195 \\
\hline & Eco1652R & CTCTACGAGACTCAAGCTTGC & \\
\hline \multirow{3}{*}{$\begin{array}{l}\text { Bacteroides } \\
\text { fragilis }\end{array}$} & g-Bfra-F & ATA GCC TTT CGA AAG & 501 \\
\hline & & RAA GAT CCA & \\
\hline & g-Bfra-R & GTA TCA ACT GCA ATT TTA & \\
\hline Clostridium & $\mathrm{g}-\mathrm{Ccoc}-\mathrm{F}$ & AAATGACGGTACCTGACTAA & 440 \\
\hline coccoides & g-Ccoc-R & CTTTGAGTTTCATTCTTGCGAA & \\
\hline Clostridium & sg-Clept-F & GCACAAGCAGTGGAGT & 239 \\
\hline leptum & sg-Clept-R & CTTCCTCCGTTTTGTCAA & \\
\hline Universal & $341-\mathrm{F}$ & CCTACGGGAGGCAGCAG & 190 \\
\hline (all bacteria) & $534-\mathrm{R}$ & ATTACCGCGGCTGCTGG & \\
\hline
\end{tabular}

2.4.7 Preparation of culture supernatant (CS) from gut bacteria. Gut bacterial suspension $\left(100 \mathrm{mg} \mathrm{mL}^{-1}\right)$ was prepared from fresh fecal samples as described recently. ${ }^{27}$ The gut bacteria were anaerobically incubated overnight in BHI medium $(20 \mathrm{mg}$ $\mathrm{mL}^{-1}$ ) at $37{ }^{\circ} \mathrm{C}$ in a GasPak EZ Anaerobe Pouch system. The bacteria were spun down by centrifugation at $3000 \mathrm{rpm}$ for $10 \mathrm{~min}$. The culture supernatant (CS) was filtered through a 0.22 $\mu \mathrm{m}$ filter and then diluted $(1: 10$ and $1: 50)$ with serum-free cell culture medium DMEM before been used for co-incubation studies according to the results of the lactate dehydrogenase $(\mathrm{LDH})$ cytotoxicity assay.

\section{$2.5 \quad$ In vitro study}

2.5.1 Direct effects of MCE and its main components on Pgp in Caco-2 cells. Caco-2 cells were cultured in DMEM containing $10 \%(\mathrm{v} / \mathrm{v})$ fetal bovine serum, $100 \mathrm{U} \mathrm{mL}^{-1}$ penicillin, and $100 \mu \mathrm{g} \mathrm{mL}^{-1}$ streptomycin. Cells were seeded into 6-well plates (Corning Inc., Cambridge, MA) at a density of $4 \times 10^{5}$ cells per well in $2 \mathrm{~mL}$ of DMEM. After 7 days, the culture medium was replaced with fresh medium or medium containing MCE $\left(0.25 \mathrm{mg} \mathrm{mL}^{-1}\right)$ or individual compound $(50 \mu \mathrm{M})$ and further incubated for $0.5,2,4,6,12$ and $24 \mathrm{~h}$, respectively, to determine the short-term effect.

To examine the long-term effects, Caco- 2 cells were seeded onto 12-well transwell ${ }^{\circledR}$ collagen-coated inserts at a density of 1 $\times 10^{5}$ cells per well and cultured in DMEM for 21 days to develop cell monolayer. Culture medium was replaced every other day. Cells were then incubated with fresh DMEM, DMEM containing MCE $\left(0.25 \mathrm{mg} \mathrm{mL} \mathrm{mL}^{-1}\right)$ or individual compound (50 $\mu \mathrm{M}$ ) for another 7 days. The culture medium was replaced with fresh DMEM or DMEM containing MCE or test compounds every two days.

2.5.2 Indirect effects of MCE on P-gp in Caco-2 cells. To determine whether Mori Cortex can modulate intestinal P-gp expression indirectly via gut bacteria-involved mechanisms, Caco-2 cells were treated with culture supernatants of gut bacteria obtained from the in vivo study. In one case, differentiated Caco-2 cell monolayers in 6-well normal plates were incubated with $\mathrm{CS}(\times 10$ and $\times 50$ dilutions $)$ of gut bacteria 
collected from normal, UC and MCE groups for $24 \mathrm{~h}$, respectively. In another case, differentiated Caco-2 cell monolayers in 12-well Transwell ${ }^{\circledR}$ plates were pre-treated with MCE $(0.25 \mathrm{mg}$ $\mathrm{mL}^{-1}$ ) for 7 days and cells cultured without MCE pretreatment served as controls. After washed with HBSS twice, the cells in MCE group and control group were incubated with CS from UC group $(\times 10$ dilution) for another $24 \mathrm{~h}$.

2.5.3 Uptake and transport of Rhodamine123 in Caco-2 cells. The short-term effect (direct effect) of MCE on P-gp activity was determined by measuring the uptake of Rho123, a probe substrate of P-gp, in Caco- 2 cells. ${ }^{31}$ After pretreated with MCE $\left(0.25 \mathrm{mg} \mathrm{mL}^{-1}\right)$ for $24 \mathrm{~h}$, cells were washed and incubated with $5 \mu \mathrm{M}$ Rho123 for 15, 30, 60, 90 and $120 \mathrm{~min}$, respectively. To determine the cellular accumulation of Rho123, $200 \mu \mathrm{L}$ of the cell lysate was added to a microplate ( 96 wells, black color, Becton-Dickinson, $\mathrm{NJ}$ ) and fluorescence intensity was measured (excitation wavelength $485 \mathrm{~nm}$, fluorescence wavelength $535 \mathrm{~nm}$ ) by SpectraMax M5 Multi-Mode Microplate Readers (Molecular Devices, USA).

The transport of Rho123 was determined to evaluate the long-term effect of MCE (direct \& indirect effect) on P-gp activity according to a previous report. ${ }^{32}$ Briefly, Caco- 2 cell monolayers in 12-well transwell plate were treated with MCE $\left(0.25 \mathrm{mg} \mathrm{mL}^{-1}\right)$ for 7 days or pretreated with MCE $(0.25 \mathrm{mg}$ $\mathrm{mL}^{-1}$ ) for 7 days followed by $24 \mathrm{~h}$-stimulation with CS from UC group $(\times 10$ dilution $)$ as described above. Samples $(100 \mu \mathrm{L})$ were taken from receiver compartments (basolateral side $1.5 \mathrm{~mL}$ or apical side $0.5 \mathrm{~mL}$ ) at 15, 30, 60, 90 and $120 \mathrm{~min}$, respectively. The fluorescence intensity of Rho123 was measured as described above. Each experiment was performed in triplicates.

\subsection{Western blotting analysis of P-gp in colon tissues and Caco-2 cells}

Colon tissues were snap frozen and ground in liquid nitrogen. Tissues or cells were lysed in presence of the protease inhibitor cocktail on ice for $30 \mathrm{~min}$. Equal amounts of lysate proteins (50 $\mu \mathrm{g}$ of colon tissue; $20 \mu \mathrm{g}$ of cells) were mixed with Laemmli sample buffer and separated in an $8 \%$ sodium dodecyl sulfate (SDS) polyacrylamide gel. The membranes were blocked in 5\% non-fat milk for $1 \mathrm{~h}$ under room temperature and incubated with monoclonal P-gp antibody (Calbiochem, Darmstadt, Germany) and GAPDH antibody in the blocking milk overnight at $4{ }^{\circ} \mathrm{C}$. Membranes were then incubated with anti-mouse IgGHRP antibody and anti-rabbit IgG-HRP antibody for $1 \mathrm{~h}$ at room temperature, respectively. The bands were visualized with Amersham ECL advanced Western blotting detection kit (GE Healthcare, Piscataway, NJ). Band intensity was analyzed with Image-Pro Plus 6.0 (IPP 6.0, Media Cybernetics, Inc., USA) software.

\subsection{Data analysis}

Data are expressed as mean \pm standard error of mean (S.E.M.). The statistical analysis was carried out using GraphPad Prism 5.0 with unpaired student's $t$-test or one way analysis of variance (ANOVA) analysis. A $P$-value less than 0.05 was deemed statistical significance. (a)

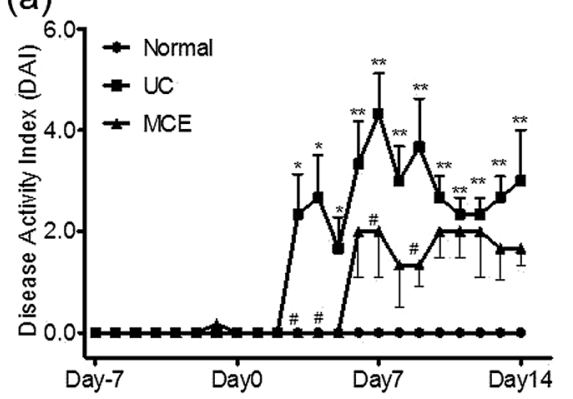

(c)

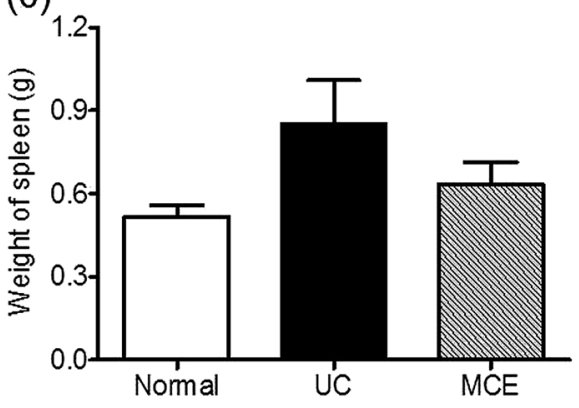

(b)

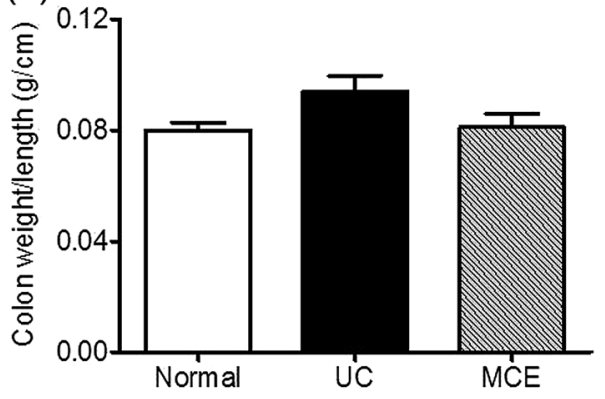

(d)

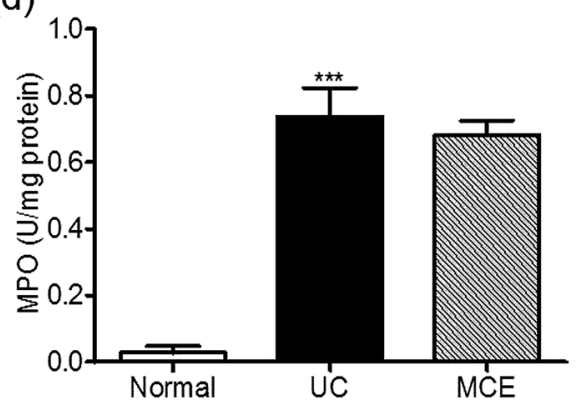

Fig. 1 MCE alleviated colitis-like symptoms in an experimental colitis rat model. (a) The disease activity index (DAI), (b) colon weight/length ratio, (c) spleen weight and (d) colon myeloperoxidase activity. Data represent the means \pm S.E.M ( $n=6$ rats per group). For induction of colitis, animals were fed with $5 \%$ DSS in drinking water for 7 consecutive days. To evaluate the effect of MCE, animals received daily oral administration of MCE $\left(0.5 \mathrm{~g} \mathrm{~kg}^{-1}\right.$ in $50 \%$ propylene glycol solution) for 3 weeks (day -7 to 14) in addition to 1 week DSS insult (day 0 to 7$)$. Normal group received drinking water only. ${ }^{*} p<0.05,{ }^{* *} p<0.01, * * * p<0.001 \mathrm{vs.} \mathrm{normal} \mathrm{group;}{ }^{*} p<0.001$ vs. UC group (ANOVA test). 


\section{Results}

\subsection{MCE alleviated DSS-induced colitis}

Rats treated with 5\% DSS developed severe colitis-like symptoms, including diarrhea, prostration, and bloody stool accompanied by severe weight loss. The disease activity index (DAI) including body weight loss, stool consistency and gross bleeding started to increase on day 3 and showed a slight decline after the abrogation of DSS (Fig. 1a). Shortening of the colon and increment of the colon weight to length ratio are typical macroscopic changes associated with DSS-induced colitis. In the present study, the DSS-treated rats still showed elevated colon weight/length ratios after DSS stimulation was halted for 1 week (day 14) (Fig. 1b). The spleen plays important roles in immune function through removing old red blood cells and synthesizing antibodies to recognize and attach foreign pathogens and allergens. Enlarged spleen (splenomegaly) is found among people with UC. ${ }^{33}$ In this study, enlarged spleens were also found in rats receiving DSS insults (Fig. 1c). The inflamed intestinal mucosa in UC is prominently featured by neutrophil accumulation. Myeloperoxidase (MPO) is most abundantly expressed in neutrophil granulocytes and its activity is taken as a quantitative measure of neutrophil accumulation influx into colon tissue. The colon mucosa collected from rats receiving DSS treatment showed significantly elevated MPO activities even after the abrogation of DSS stimulation (Fig. 1d). Histological assessment of the distal colon tissues revealed inflammatory cell infiltration in the mucosa, loss of goblet cells
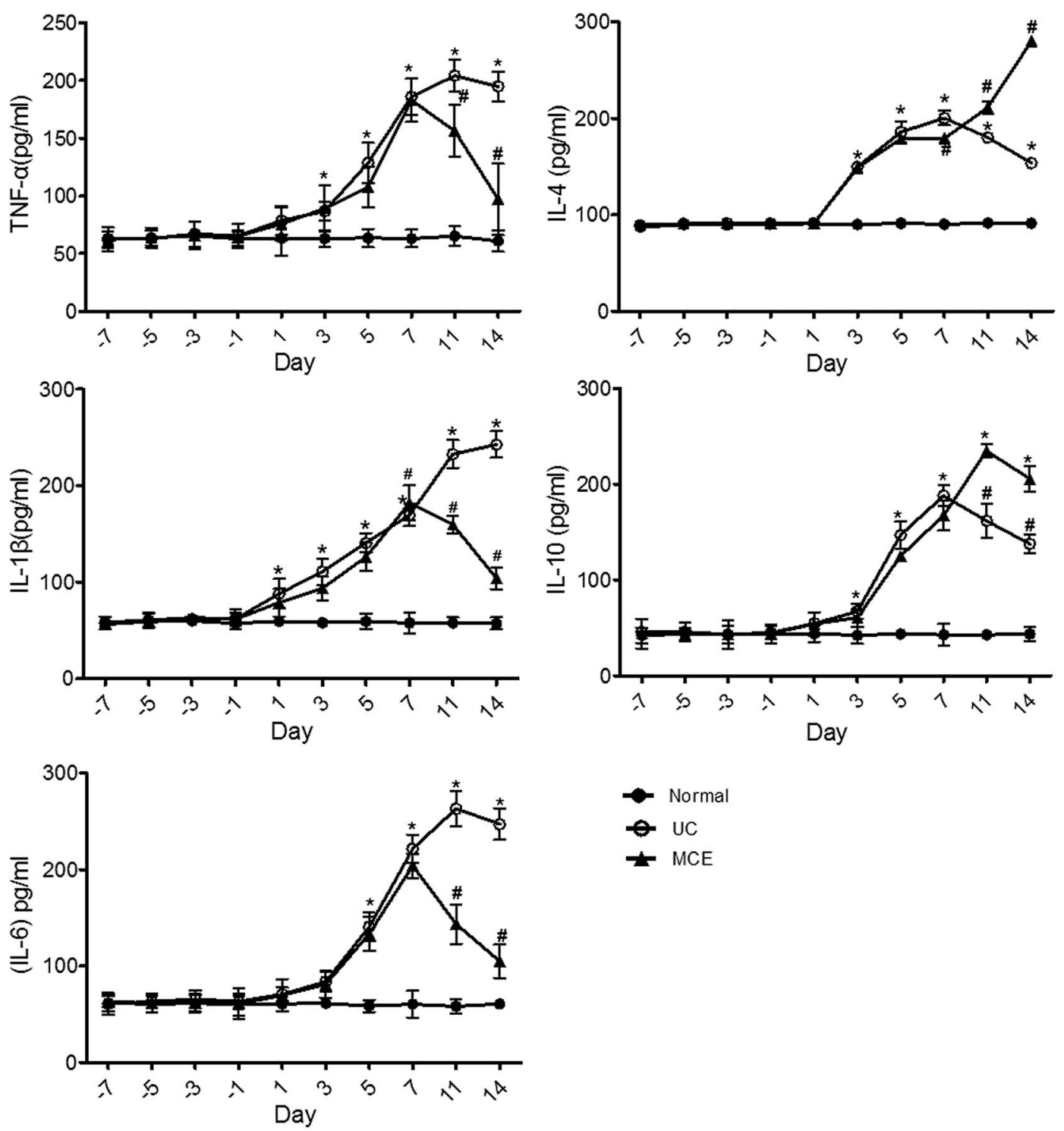

Fig. 2 MCE treatment altered the cytokine profile in rats with DSS-induced colitis. The concentrations of TNF- $\alpha$, IL-1 $\beta$, IL-6, IL-4, and IL-10 in serum were measured with ELISA kits. Data represent means \pm S.E.M ( $n=6$ rats per group). For induction of colitis, animals were fed with $5 \%$ DSS in drinking water for 7 consecutive days. To evaluate the effect of $\mathrm{MCE}$, animals received daily oral administration of $\mathrm{MCE}\left(0.5 \mathrm{~g} \mathrm{~kg}{ }^{-1}\right.$ in $50 \%$ propylene glycol solution) for 3 weeks (day -7 to 14 ) in addition to 1 week DSS insult (day 0 to 7). Normal group received drinking water only. * $p<$ 0.05 vs. normal group, ${ }^{\#} p<0.05$ vs. UC group (ANOVA test). 
and epithelium, distorted crypts, and edema in the rats with DSS-induced colitis (ESI Fig. S1†).

Daily oral intake of the MCE for continuous 3 weeks attenuated the responses of animals to DSS stimulation, leading to decreased DAI scores (Fig. 1a) and lower colon weight/length ratios (Fig. 1b) than the UC group. MCE treatment also alleviated spleen enlargement caused by DSS (Fig. 1c). The colonic mucosa from MCE treated rats showed histological improvement in crypt architecture, reduction in the levels of mucosal injury, edema, and infiltration of inflammatory cells (ESI Fig. S1†). However, the MPO activity in colon mucosa, which was significantly elevated by DSS, was unaltered by MCE treatment (Fig. 1d).

\subsection{MCE attenuated the inflammation in rats with colitis}

The cytokine network in IBD is a complex, dynamic system that plays an important role in disease pathogenesis and progression. ${ }^{34}$ In the acute experimental colitis rat model induced by DSS, both pro-(TNF- $\alpha$, IL-1 $\beta$, IL-6) and anti-inflammatory (IL-4 and IL-10) cytokines were significantly increased after DSS stimulation and showed a time-dependent manner (Fig. 2). Except for IL-4 which was detected on day 3, all other cytokines were detected on day 1 after initiation of DSS stimulation. After DSS withdrawal, the pro-inflammatory cytokine levels kept increasing, although at slower rates, and reached maximum levels on day 11 (TNF- $\alpha$, IL-6) or day 14 (IL-1 $\beta$ ), while the levels of both anti-inflammatory cytokines (IL-4 and IL-10) were significantly decreased, which on day 14 was yet much higher than the basal levels. The pattern of cytokines supports the acute selflimited character of ulcerative colitis, ${ }^{34}$ however, the everincreasing pro-inflammatory cytokines observed in DSStreated rats were different from those reported in the DSSinduced mice colitis model in which both the anti-(IL-10 and IL-12) and the pro-inflammatory cytokines followed bi-phasic changes: increase with DSS stimulation and decrease after DSS withdrawal. ${ }^{26}$

In general, the 1 week MCE pretreatment of rats prior to initiating the DSS stimulation did not block the cytokines elevation elicited by DSS insult. However, concomitant daily oral intake of MCE could mitigate the further increases of the pro-inflammatory cytokines, which were close to the basal levels on day 14, while enhanced the production of anti-inflammatory cytokines in the recovery phase (Fig. 2).

\subsection{MCE abrogated colonic P-gp decrease in DSS rat colitis}

Compared to normal rats, the rats of UC group showed a significantly decreased P-gp expression $(\sim 60 \%)$ in colon mucosa on day 14 (Fig. 3). Interestingly, the MCE treatment abrogated the decrease of P-gp protein expression induced by DSS, leading to the P-gp protein level comparable to the normal group.

\subsection{MCE altered microbial composition in colitic rats}

In our study, the total bacteria and 5 bacteria which have been reported to be associated with UC were measured with the developed 16S rRNA-based RT-PCR method (Fig. 4). The

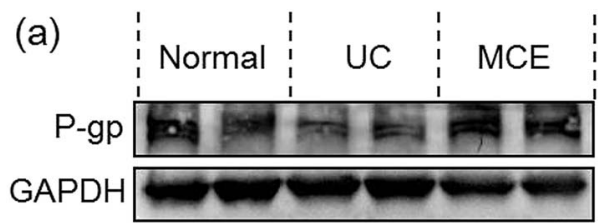

(b)

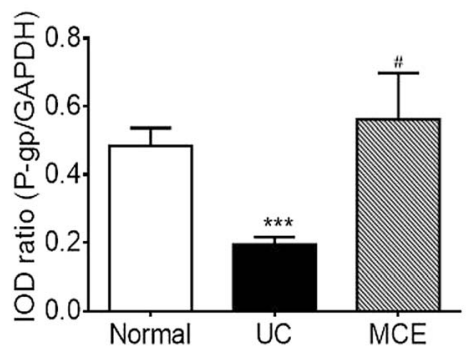

Fig. 3 MCE abrogated colonic P-gp decrease in DSS rat colitis. The Pgp expression in colon tissue was evaluated using western blot and the membranes were probed with antibody against P-gp and GAPDH, respectively. (a) Representative western blot stain; (b) relative stain intensity. Data represent means \pm S.E.M ( $n=6$ rats per group). For induction of colitis, animals were fed with $5 \%$ DSS in drinking water for 7 consecutive days. To evaluate the effect of MCE, animals received daily oral administration of MCE $\left(0.5 \mathrm{~g} \mathrm{~kg}^{-1}\right.$ in $50 \%$ propylene glycol solution) for 3 weeks (day -7 to 14 ) in addition to 1 week DSS insult (day 0 to 7). Normal group received drinking water only. ${ }^{* * *} p<0.001$ vs. normal group, ${ }^{\#} p<0.05$ vs. UC group (ANOVA test).

amount of the total bacteria in normal group was relatively stable throughout the experimental period. DSS stimulation resulted in a quick decrease of the total bacteria which was restored on day 7. On day 1, the genome copies of Enterobacteriaeae and Bacteroides fragilis in UC group showed slight increases with significant differences observed in Enterobacteriaeae content, while the genome copies of Clostridium coccoides were decreased, and Clostridium leptum and Bifidobacterium were unaltered by DSS stimulation. When DSS stimulation was continued, rats of UC group showed significant decreases in Bifidobacterium and Clostridium coccoides on day 7. The level of Clostridium leptum didn't show significant changes. The Enterobacteriaeae and Bacteroides fragilis were significantly higher than that of the normal group and 5-20 folds that of respective initial levels determined on day 7. After removal of DSS stimulation, rats of the UC group showed a complete restoration of Enterobacteriaceae and a partial restoration of Bacteroides fragilis, while the genome copies of Bifidobacterium and Clostridium coccoides retained at decreased levels on day 14. The level of Clostridium leptum remained the same to normal group on day 7 , while a significant decrease was observed one week after withdrawal of DSS (day 14).

In the MCE group, the genome copies of beneficial bacteria Bifidobacterium were increased by MCE treatment which was significantly higher than both normal and UC groups. In contrast, the genome copies of Enterobacteriaceae and Bacteroides fragilis enhanced by DSS stimulation were completely counteracted by concomitant oral administration of MCE. However, the MCE treated rats exhibited marked decreases in the predominant gut bacteria Clostridium coccoides and Clostridium leptum at the early stage of DSS insult (day 1), which 

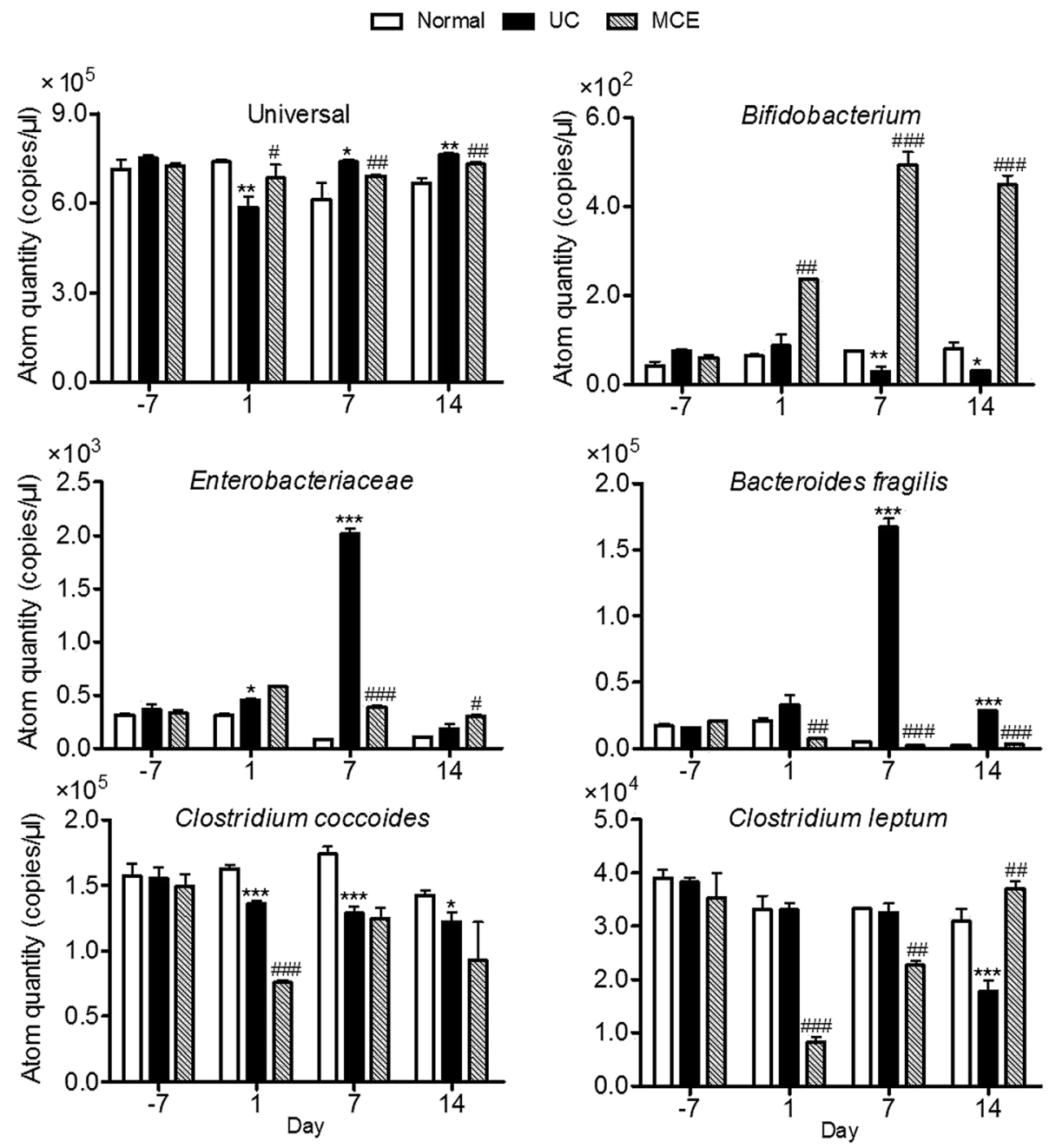

Fig. 4 MCE altered microbial composition in DSS-treated rats. Fresh fecal samples collected from each rat of each group were pooled and prepared for fecal bacteria. DNA from fecal bacteria was extracted using MiniBEST bacterial genomic DNA extraction kit. Then fecal DNA was assayed immediately using $16 \mathrm{~S}$ rRNA RT-PCR. Data represent means \pm S.E.M. from triplicate reactions. For induction of ulcerative colitis, animals were fed with 5\% DSS in drinking water for 7 consecutive days from day 0 to 7 . To evaluate the effect of MCE, animals received 3 week (day -7 to day 14) MCE intervention (50\% propylene glycol solution, oral gavage) plus 1 week DSS insult. $* p<0.05, * * p<0.01, * * * p<0.001$ vs. normal group; ${ }^{\#} p<0.05,{ }^{\# \#} p<0.01,{ }^{\# \# \#} p<0.001$ vs. UC group (ANOVA test).

partially restored on day 7 (Clostridium coccoides) or completely restored to normal level on day 14 (Clostridium leptum).

\subsection{MCE regulated P-gp in Caco-2 cells in a biphasic manner}

A concentration of $250 \mu \mathrm{g} \mathrm{mL}^{-1}$ of MCE was chosen for the in vitro experiments according to the results of cytotoxicity and anti-proliferation assays on Caco- 2 cells. When Caco- 2 cells were treated by MCE for a short time period (up to $24 \mathrm{~h}$ ), as shown in Fig. 5a, the P-gp expression was inhibited in a timedependent manner with a $67 \%$ inhibition at $24 \mathrm{~h}$. However, an opposite effect was observed when MCE pretreatment was extended to 7 days (Fig. 5b), indicating a biphasic regulation of MCE on intestinal P-gp expression.

The biphasic regulation effect of MCE on P-gp in Caco-2 cells was further consolidated with Rho123 uptake and transport studies. As shown in Fig. 5c, the accumulation of Rho123 in Caco- 2 cells increased in a time-dependent manner within $24 \mathrm{~h}$ of incubation. The presence of MCE significantly enhanced the uptake of Rho123 into Caco- 2 cells by $46-69 \%$ at all time points tested, agreeing well with the decreased P-gp expression detected in MCE-treated Caco-2 cells. Rho123 showed a preferential transport in the BL-to-AP direction which was further enhanced by the long-term treatment (7 days) of Caco- 2 cells with MCE, resulting in the mean efflux ratio of Rho123 1.4-fold of control group (Fig. 5d). This enhanced efflux of Rho123 by long-term treatment with MCE is in concert with the upregulation of P-gp expression in Caco- 2 cells.

\subsection{MCE constituents regulated P-gp expression differently}

As shown in ESI Fig. S3a, $\uparrow 24$ hour exposure to mulberroside A and sanggenon $\mathrm{C}$ resulted in significant induction of P-gp expression in Caco-2 cells, while morusin led to a downregulation of P-gp expression. Kuwanon G, morin, and oxyresveratrol showed no effects within 24 hour. When the 
(a)

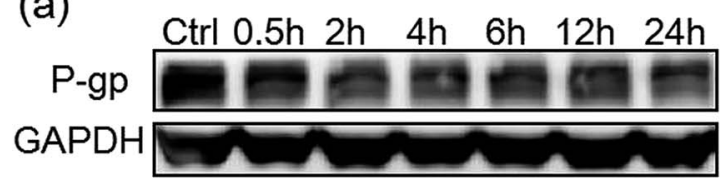

(b)

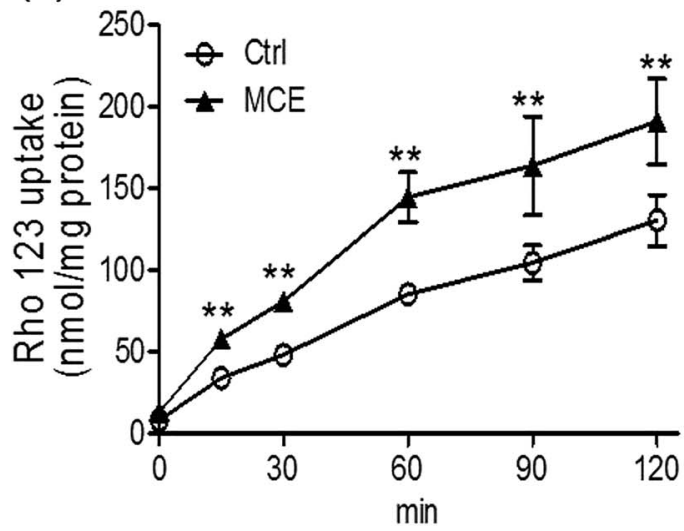

(c)

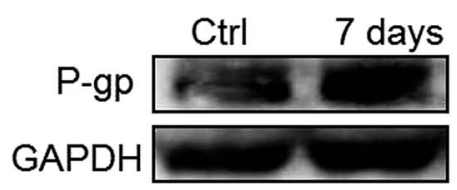

(d)

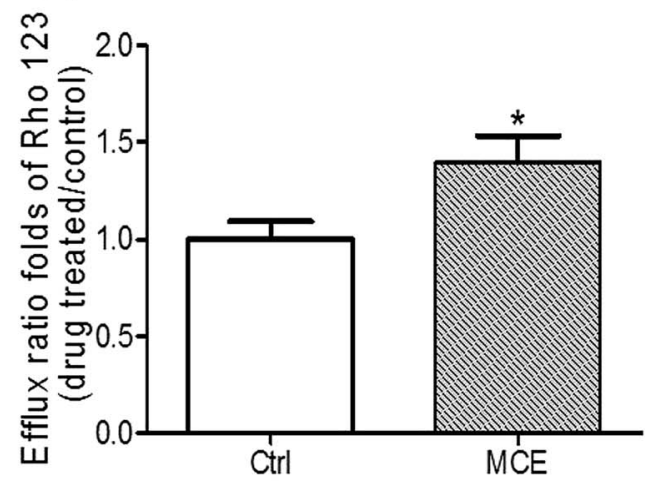

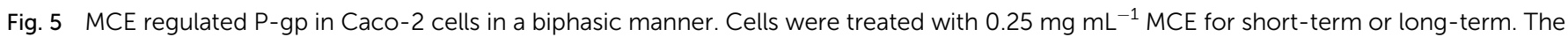
short-term effect (up to 24 h) on P-gp was evaluated using Western blot (a) and uptake of Rho123 (b); the long-term effect (7 days) on P-gp was evaluated using western blot (c) and transport of Rho123 (20 $\mu \mathrm{M})(\mathrm{d})$ across Caco-2 cell monolayers. Cells incubated with medium in parallel for $0 \mathrm{~h}$ (short-term) and 7 days (long-term) served as controls. Data represented mean \pm S.E.M. from triplicate experiments. ${ }^{*} p<0.05$ vs. control (Ctrl) group, ${ }^{* *} p<0.01$ vs. Ctrl group (unpaired student's $t$-test).

incubation time was prolonged to 7 days (ESI Fig. S3b†), P-gp expression in presence of sanggenon $\mathrm{C}$, kuwannon $\mathrm{G}$, morin and mulberroside A was up-regulated, while 7 day exposure to morusin did not affect P-gp expression significantly. Oxyresveratrol still showed no effect on P-gp expression in Caco-2 cells when the exposure was extended.

\subsection{MCE modulated P-gp in Caco-2 cells via gut bacteria- involved mechanism}

To evaluate the involvement of gut bacteria on regulation of P-gp, Caco-2 cells were treated with the culture supernatant (CS) of gut bacteria from different groups for $24 \mathrm{~h}$. As shown in Fig. 6a and b, P-gp protein levels in Caco- 2 cells were decreased by CS from the UC group with a significant change ( $\sim 2$-fold) observed with the CS of 10-fold dilution. It is interesting to note that the CS from MCE group showed the tendency to enhance Pgp expression in Caco-2 cells at two dilution levels when compared with the cells treated by CS from the normal group, although there was no statistically significant difference (Fig. 6b). Pretreatment of the Caco-2 cells with MCE for 7 days aborted the down-regulation of P-gp expression by the CS from UC group (10-fold dilution) (Fig. 6d), leading to an enhanced transport of Rho123 in the secretory direction (efflux ratio $\sim 1.2$ folds of the control group) (Fig. 6e), indicating that long-term pretreatment with MCE could prevent the decrease of P-gp activity by CS from UC group.

\subsection{CS from different groups stimulated cytokines production in Caco-2 cells}

The production of pro-inflammatory (TNF- $\alpha$, IL-1 $\beta$, IL- 6 and IL8) and anti-inflammatory (IL-4 and IL-10) cytokines in Caco-2 cells treated by CS (10-fold dilution) from different groups are shown in Fig. $6 \mathrm{c}$ and f. IL-4 and IL-6 were not detected in culture medium of Caco- 2 cells in all cases. The CS from both normal and UC groups stimulated the production of minor TNF- $\alpha$ and IL-1 $\beta$ and high amounts of IL- 8 and IL-10 in Caco- 2 cells. Cells exposed to normal CS generated IL-10 in the highest amount, while that treated by CS from UC rats yielded significantly higher amounts of IL-8 and IL-1 $\beta$ and a lower IL-10 level than the normal CS treated cells. The exposure of Caco- 2 cells to the CS from MCE-treated UC rats (MCE group) resulted in production of IL-1 $\beta$ and IL- 8 comparable to the normal group, whereas, the IL-10 generated was significantly higher than the UC CS and even higher than that produced by cells stimulated by the normal CS.

Similarly, when the Caco- 2 cells were pretreated with MCE for one week, as shown in Fig. 6f, the induction of proinflammatory cytokines, in particular IL-8, by CS from UC group (10-fold dilution) was significantly inhibited. However, the production of the anti-inflammatory cytokine IL-10 induced by CS from UC group was unaltered by MCE pretreatment.

\section{Discussion}

The present study revealed, for the first time, a protective role of Mori Cortex, a traditional Chinese medicine, in an experimental colitis induced by DSS in rats. MCE administration alleviated UC symptoms accompanying with an improvement of immune functions, an enhancement of the intestinal epithelial integrity (colon P-gp up-regulation) and a restoration of microbial balance. Further in vitro studies in Caco- 2 cells implicated the involvement of gut bacteria in diminished P-gp expression in 


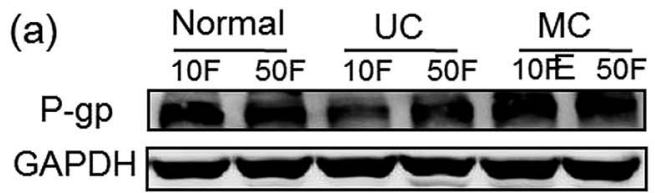

(b)

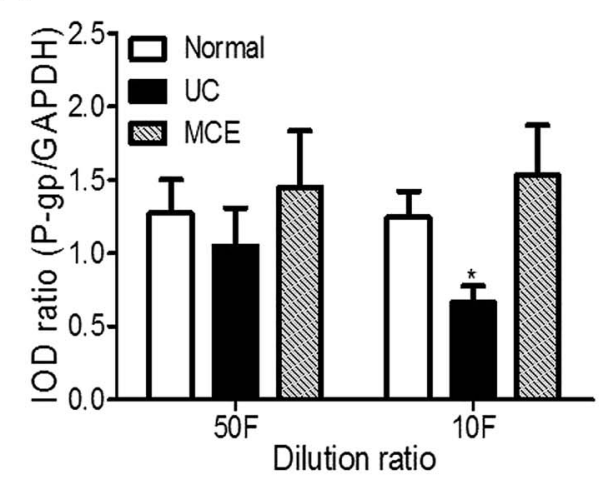

(c)

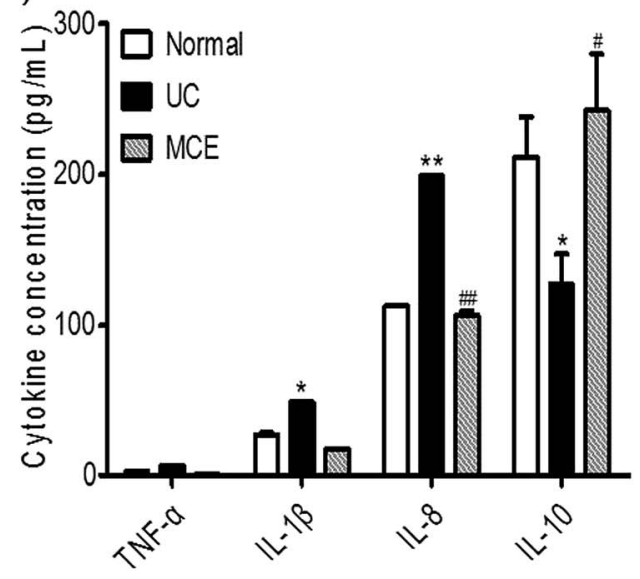

(d)

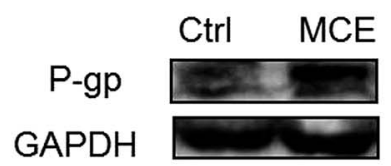

(e)

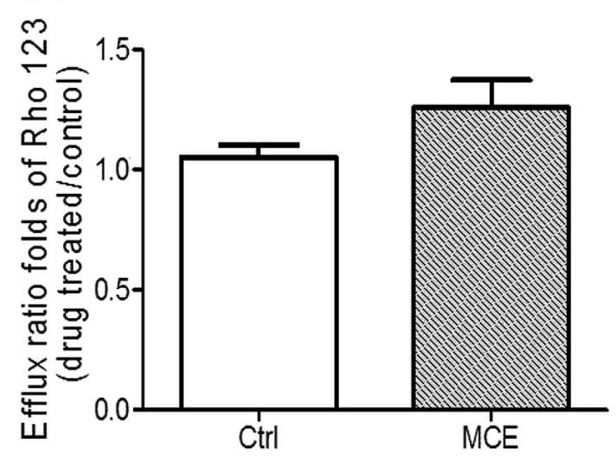

(f)

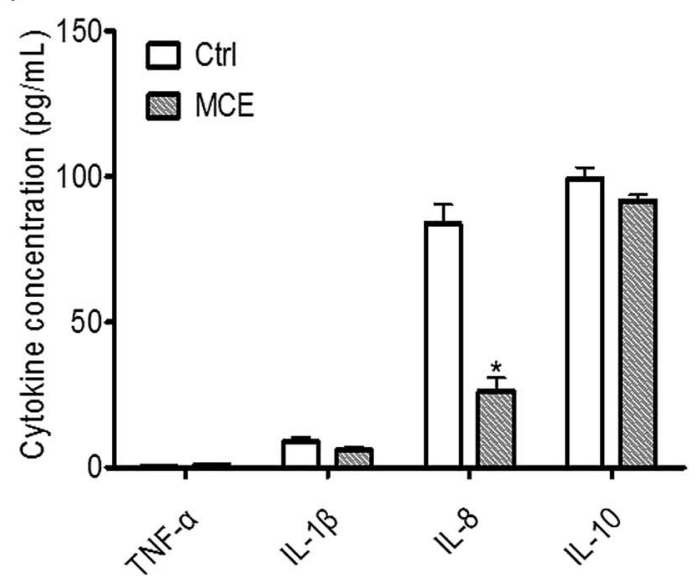

Fig. 6 MCE modulated P-gp in Caco-2 cells via gut bacteria-involved mechanism. Effect of CS of gut bacteria from normal, UC and MCE groups on P-gp expression ((a) representative western blot stain; (b) relative stain intensity) and inflammatory mediators production (c) in Caco-2 cells; effects of MCE pretreatment on P-gp expression ((d) representative Western blot stain) and function ((e) Rho123 transport) and cytokine levels (f) in Caco-2 cells treated by CS from colitis rats. Data represent means \pm S.E.M. from triplicate reactions. CS means culture supernatant. * $p<0.05$, $* * p<0.01$ vs. normal group; ${ }^{*} p<0.05$ vs. UC group (ANOVA test and unpaired student's $t$-test).

DSS-induced acute colitis and its contribution to the beneficial activity of Mori Cortex observed in the animal model.

Key features of UC include diffuse mucosal inflammation that extends proximally from the rectum to a varying degree. An increasing amount of experimental evidences indicated that DSS-colitis is mainly associated with innate immune mechanisms and is thought to resemble human UC. In this study, the DSS-induced rat colitis model exhibited the symptoms (body weight loss, diarrhea, bloody feces, mucosal ulceration etc.) similar to human UC. The elevated MPO activity in the colon mucosa demonstrated neutrophils infiltration in colonic tissue. The spleen enlargement (splenomegaly) which has been reported in human UC was also observed in DSS-induced rat colitis. Dynamic serum cytokines profiling revealed that DSS stimulation evoked earlier production of pro-inflammatory cytokines (IL-1 $\beta$, TNF-a, IL-6) and the anti-inflammatory cytokine IL-4 than IL-10 in rats. This data agreed with the finding of a recent study which reported earlier elevation of TNF- $\alpha$ and IL-1 $\beta$ than IL-10 in mice with DSS-induced colitis. ${ }^{50}$ These anti-inflammatory cytokines act synergistically in the down-regulation of pro-inflammatory cytokines, leading to a significant improvement of inflammatory symptoms. ${ }^{34}$ These findings support the DSS-induced rat colitis model is suitable for studying human UC. The extract of Mori Cortex could alleviate the colitis symptoms. However, it did not significantly decrease MPO activity or block the production of the proinflammatory cytokines during DSS stimulation. Oppositely, the herb can suppress the production of pro-inflammatory cytokines while enhance the generation of anti-inflammatory cytokines after DSS withdrawal. These results were consistent with the findings in vitro that the soluble factors from gut bacteria of MCE group decreased the production of IL- $1 \beta$ and 
IL-8 but enhanced IL-10 generation and MCE-pretreated Caco-2 cells showed significantly decreased IL-8 generation.

Decreased P-gp function and expression in inflamed intestinal epithelium have been implicated in the pathogenesis of $\mathrm{IBD}^{7}$ and the gene $M D R 1$ has been proposed as a candidate gene for IBD therapy. P-gp has a wide substrate affinity and thus is believed to act as a cytotoxic protection. In addition, elevated Pgp expression decreases bacterial association with human gastrointestinal cells. ${ }^{35}$ P-gp dysregulation preceded in the large intestine before intense inflammatory symptoms appeared in DSS-induced colitis mice model, ${ }^{36}$ indicating that P-gp downregulation is one of the early events in colitis progression. In the present study, a diminished P-gp expression in colon tissues of rats with DSS-induced colitis was consolidated, which was in agreement with the finding in DSS-induced colitis mice model ${ }^{36}$ and TNBS-induced colitis rat in rats. ${ }^{37}$ In this study, we first demonstrated that MCE abrogated the P-gp down-regulation by DSS stimulation, suggesting that enhancement of the intestinal epithelial integrity might contribute to the observed beneficial effects of MCE on DSS-induced colitis in rats.

Another common feature of IBD is microbial imbalance. The reduction of total microbial diversity, increases of Enterobacteriaceae, Bacteroides-Prevotella, and decrease of Firmicutes were found in IBD patients. ${ }^{38}$ These microbial alterations were accompanied with the immune imbalance and are believed to play a crucial role in inflammatory events in IBD. In our study, using the 16S rRNA-based PCR analysis, we determined the changes of total gut bacteria and 5 specific bacteria that have been reported to be implicated in UC patients. ${ }^{39}$ In responses to DSS insult, the beneficial bacteria Bifidobacterium, which could utilize glucose and lactose to produce lactic acid inhibiting pathogens and spoilage organisms in the intestines, ${ }^{\mathbf{4 0}}$ were decreased in rats, agreeing with previous clinical studies. ${ }^{\mathbf{4 1}}$ However, another study reported the increases of Bifidobacterium group in active IBD patients. ${ }^{42}$ In addition to the different status of IBD of the patients and the colitis rat model, these conflicting results might also be evoked by the lower specificity of primer used for quantitative PCR of Bifidobacterium than other genus. ${ }^{42}$ Furthermore, different Bifidobacterium may act differently in IBD. Thus, it is necessary to combine 16S rRNAbased RT-PCR analysis using the sub-group specific primers of Bifidobacterium plus denatured gradient gel electrophoresis (DGGE) profiling to further identify the species that is altered by DSS-induced colitis in rats and by Mori Cortex. A recent study indicated that probiotics exert their protective effects by increasing the reduced expression of P-gp in intestinal inflammation. ${ }^{15}$ Thus, the beneficial effects of Mori Cortex extract on DSS-induced rat colitis may be partly attributed to the stimulation on Bifidobacterium growth.

Elevation of the opportunistic pathogen including Enterobacteriaceae and Bacteroides fragilis has been reported in IBD patients, and both are selected as candidate markers of gut microbiota for IBD. ${ }^{39,43}$ These two bacteria displayed significant increases in responses to DSS insult (day 7) which, however, were restored after DSS was withdrawn (day 14). The biphasic alteration of Enterobacteriaceae and Bacteroides fragilis fit the acute self-limited feature of 7 day DSS-induced rat colitis. Daily oral administration of Mori Cortex extract prevented the significant increases of these opportunistic pathogens elicited by DSS.

Both Clostridium leptum and Clostridium coccoides belong to genus Clostridium, one of the dominant groups of fecal microbiota in adult humans including IBD patients. ${ }^{44}$ They were used as the directive bacteria to evaluate the whole state of inflammatory processes of intestinal diseases and were reduced both in active disease and during disease remission of IBD patients in clinical studies. ${ }^{38,45}$ In the rat colitis model, Clostridium coccoides showed a gradual decrease throughout the acute and recovery phases, while Clostridium leptum only showed an abrupt decrease on day 14. However, in MCE-treated rats, both bacteria groups showed significant decreases after 1 day DSS insult although a restoration was observed on day 7 or day 14 . Since both Clostridium leptum and Clostridium coccoides contain a large number of butyrate-producing bacteria which play an important role in the human colon through supplying energy to the gut epithelium and regulating host cell responses, the rapid and reversible inhibition of MCE on the two predominant gut bacterial groups warrants further study to evaluate the safety of the herbal medicine in alleviation of UC.

Numerous data from previous studies have linked the P-gp dysregulation in IBD to the altered immune balance. Gut microbiota play an important role in intestinal mucosal immunity. ${ }^{42}$ The altered microbial composition (gut dysbiosis) may result in changes of host-gut microbiota co-metabolites and microbial secondary metabolites which disturb intestinal mucosal homeostasis, leading to disruption of the intestinal barrier and/or enhance a host inflammatory response and thereby, aggravate the intestinal inflammation. ${ }^{46}$ Mass spectrometry analysis of the soluble factors (culture supernatant) of gut microbiota from DSS-induced UC rats showed altered nutrition utilization and metabolic profile (our group unpublished data). The microbial soluble factors from UC rats caused downregulation of P-gp expression and function in Caco- 2 cells. In a recent study, soluble factors from probiotic Lactobacilli upregulated P-gp expression in Caco-2 cells and blocked the reduced expression of $m d r 1 a / \mathrm{P}$-gp mRNA and protein in the distal colon in DSS-treated mice. ${ }^{15}$ Taking together, these findings provided insights into a gut microbiota-mediated P-gp regulatory mechanism and new therapeutic strategies for UC. In this study, we speculated that the colon P-gp up-regulation by MCE involve both direct effects and gut microbiota-mediated mechanisms and examined it in vitro on Caco- 2 cells. We observed direct biphasic effects of MCE on P-gp expression in Caco- 2 cells with down-regulation in short-term (up to $24 \mathrm{~h}$ ) and up-regulation in long-term (up to 7 day) exposures to MCE. This time-dependent modulatory effects of MCE on P-gp expression can be partially explained by the varied effects of the main components in the extract under short- and long-term treatments (ESI Fig. S3†). Indeed, a number of studies have shown that the modulation effects on P-gp were essentially affected by incubation time. For instance, short/long-term exposure to NO donors led to different regulation effects on P-gp expression and activity in Caco-2 cells via NO-independent mechanisms. ${ }^{47}$ Similar biphasic patterns were also found for P-gp in rat brain 
capillaries exposed to TNF- $\alpha$ and endothelin- $1 .{ }^{48}$ However, since 7 day cells and 21 day cell monolayers were adopted in this study for studying the short- and long-term effects, respectively, we could not rule out the possibility that cells at different states may respond differently to the same stimuli. Taken together, it can be concluded that the pattern of P-gp expression and activity in response to MCE was complex, and the up-regulation of P-gp by MCE in the colons of colitic rats was partially due to the long-term direct effect of MCE treatment. Further studies are warranted to elucidate whether the short- and long-term effects are signaled through a common pathway.

Several studies have implicated the associations between gut dysbiosis and the diminished P-gp expression and activity in the pathogenesis of intestinal inflammation. In this study, we demonstrated that the soluble factors secreted by gut microbiota from DSS-treated rats can suppress P-gp expression and alter cytokines production (enhanced IL-1 $\beta$ and IL-8 and suppressed IL-10) which, however, can be abrogated by oral administration of Mori Cortex extract to rats or pretreatment of Caco-2 cells with the extract. So far, several types of soluble macromolecules or small molecules, such as proteins, ${ }^{\mathbf{4 9}}$ shortchain fatty acids (acetate, propionate, butyrate), ${ }^{50}$ bacteriocins $^{51}$ have been identified from intestinal bacteria and some were demonstrated to profoundly influence gut barrier function, host immunity, epithelial proliferation and bacterial pathogenesis. ${ }^{52}$ A previous report ${ }^{53}$ and our unpublished data found that co-incubation of Caco- 2 cells with mucosal bacteria from ulcerative colitis patients resulted in similar changes in Pgp expression and cytokines production. Although we were not able to provide unambiguous evidence on gut bacteriamediating intestinal P-gp modulation in the current study, the accumulating knowledge on the interplays between gut microbiota and mucosal immunity, and intestinal mucosal immunity and drug transport system allow us to offer the following speculation: microbial imbalance elicited by DSS resulted in altered microbial products or host-microbial cometabolites, which in turn down-regulate colon P-gp expression directly or through stimulating production of some cytokines, for instance, IL-8 which was reported to decrease P-gp in Caco-2 cells. ${ }^{54}$

Previous studies have demonstrated a marked antiinflammatory effect of Mori Cortex. It's interesting to note that MCE showed insignificant effect on elevated MPO activity, while inhibited pro-inflammatory cytokines production and enhanced anti-inflammatory cytokines production in DSStreated rats. This cytokine production profile was in good agreement with that observed in Caco- 2 cells treated by soluble factors of gut bacteria from different groups. Moreover, the pretreatment of the Caco- 2 cells with MCE following by incubation with CS from UC rats alleviated the inflammatory responses, reversed the down-regulation of P-gp expression in Caco-2 cells treated by CS from UC rats alone. These data imply the involvement of gut microbiota in the regulatory effects of MCE on P-gp expression. By applying several main constituents in the herbal extract on Caco-2 cells, we observed direct effects of these compounds on P-gp expression which could partially explain the biphasic regulatory effects of MCE on P-gp. A recent study investigated mulberroside A on P-gp modulation and found mulberroside A could down-regulate P-gp expression and function in both Caco-2 cells and normal rats as well as activate protein kinase $\mathrm{C}(\mathrm{PKC})$ and NF- $\mathrm{KB}$ in Caco-2 cells. ${ }^{55}$

\section{Conclusion}

In summary, P-gp expression was decreased in colon tissues of rats with experimental colitis induced by DSS. Mori Cortex extract markedly alleviated the inflammatory responses and enhanced P-gp-mediated intestinal epithelial integrity. In vivo and in vitro studies indicated that the regulatory effects of Mori Cortex on colon P-gp may involve both direct and indirect mechanisms which highlight the potential of P-gp or gut microbiota as new therapeutic target for IBD therapy. Our ongoing work aims to consolidate the P-gp regulatory effects of gut microbiota and identify the main bacterial strains involved and molecular events using germ-free animal models.

\section{Acknowledgements}

This work is financially supported by the Science and Technology Development Fund of Macao SAR (Ref. No. 029/2015/A1, 043/2011/A2), the National Basic Research Program of China 973 program (Grant No. 2009CB522707), the National Natural Science Foundation (Ref. no.: 81473281), and the University of Macau (Ref. no. MYRG 2015-00220-ICMS-QRCM).

\section{References}

1 M. Camilleri, K. Madsen, R. Spiller, B. Greenwood-Van Meerveld and G. N. Verne, Neurogastroenterol. Motil., 2012, 24, 503-512.

2 P. Henderson, J. E. van Limbergen, J. Schwarze and D. C. Wilson, Inflammatory Bowel Dis., 2011, 17, 382-395.

3 K. J. Maloy and F. Powrie, Nature, 2011, 474, 298-306.

4 R. Silva, V. Vilas-Boas, H. Carmo, R. J. Dinis-Oliveira, F. Carvalho, M. de Lourdes Bastos and F. Remiao, Pharmacol. Ther., 2015, 149, 1-123.

5 R. W. Johnstone, A. A. Ruefli and M. J. Smyth, Trends Biochem. Sci., 2000, 25, 1-6.

6 L. Kagan, T. Dreifinger, D. E. Mager and A. Hoffman, Drug Metab. Dispos., 2010, 38, 1560-1566.

7 G. Englund, A. Jacobson, F. Rorsman, P. Artursson, A. Kindmark and A. Ronnblom, Inflammatory Bowel Dis., 2007, 13, 291-297.

8 M. Fakhoury, J. Lecordier, Y. Medard, M. Peuchmaur and E. Jacqz-Agrain, Inflammatory Bowel Dis., 2006, 12, 745-749.

9 M. Brinar, S. Cukovic-Cavka, N. Bozina, K. G. Ravic, P. Markos, A. Ladic, M. Cota, Z. Krznaric and B. Vucelic, BMC Gastroenterol., 2013, 13, 57.

10 K. H. Banner, C. Cattaneo, J. L. Le Net, A. Popovic, D. Collins and J. D. Gale, Br. J. Pharmacol., 2004, 143, 590-598.

11 X. Y. Chu, J. R. Strauss, M. A. Mariano, J. Li, D. J. Newton, X. Cai, R. W. Wang, J. Yabut, D. P. Hartley, D. C. Evans and R. Evers, J. Pharmacol. Exp. Ther., 2006, 317, 579-589. 
12 K. Matsuoka and T. Kanai, Semin. Immunopathol., 2015, 37, 47-55.

13 K. Nones, B. Knoch, Y. E. Dommels, G. Paturi, C. Butts, W. C. McNabb and N. C. Roy, J. Appl. Microbiol., 2009, 107, 557-566.

14 C. M. Panwala, J. C. Jones and J. L. Viney, J. Immunol., 1998, 161, 5733-5744.

15 S. Saksena, S. Goyal, G. Raheja, V. Singh, M. Akhtar, T. M. Nazir, W. A. Alrefai, R. K. Gill and P. K. Dudeja, Am. J. Physiol.: Gastrointest. Liver Physiol., 2011, 300, G1115G1123.

16 H. Z. Toklu, L. Kabasakal, N. Imeryuz, B. Kan, C. Celikel, S. Cetinel, O. Orun, M. Yuksel and G. A. Dulger, J. Physiol. Pharmacol., 2013, 64, 439-451.

17 S. H. Zheng and R. T. Cui, Shanghai Journal of Traditional Chinese Medicine, 2000, 34, 3.

18 Y. C. Chen, Y. J. Tien, C. H. Chen, F. N. Beltran, E. C. Amor, R. J. Wang, D. J. Wu, C. Mettling, Y. L. Lin and W. C. Yang, BMC Complementary Altern. Med., 2013, 13, 45.

19 A. Ahmad, G. Gupta, M. Afzal, I. Kazmi and F. Anwar, Life Sci., 2013, 92, 202-210.

20 W. Wang, Y. G. Zu, Y. J. Fu and T. Efferth, Am. J. Chin. Med., 2012, 40, 349-356.

21 M. A. Abdulla, H. M. Ali, K. A. Ahmed, S. M. Noor and I. Salmah, Biomed. Res., 2009, 20, 35-39.

22 P. W. Hsu, C. S. Shia, S. P. Lin, P. D. Chao, S. H. Juang and Y. C. Hou, J. Agric. Food Chem., 2013, 61, 4464-4469.

23 A. I. Alvarez, R. Real, M. Perez, G. Mendoza, J. G. Prieto and G. Merino, J. Pharm. Sci., 2010, 99, 598-617.

24 M. T. Sheu, Y. B. Liou, Y. H. Kao, Y. K. Lin and H. O. Ho, Chem. Pharm. Bull., 2010, 58, 1187-1194.

25 W. H. Jing, Y. Ru and Y. T. Wang, Anal. Methods, 2015, 7, 443-457.

26 Y. J. Huang, N. Hu, X. J. Gao, Z. X. Yan, S. Li, W. H. Jing and R. Yan, Chem.-Biol. Interact., 2015, 232, 38-48.

27 R. N. Zhou, Y. L. Song, J. Q. Ruan, Y. T. Wang and R. Yan, Drug Metab. Pharmacokinet., 2012, 27, 586-597.

28 J. Q. Ruan, S. Li, Y. P. Li, W. J. Wu, S. M. Lee and R. Yan, Drug Metab. Dispos., 2015, 43, 1601-1611.

29 P. S. Langendijk, F. Schut, G. J. Jansen, G. C. Raangs, G. R. Kamphuis, M. H. Wilkinson and G. W. Welling, Appl. Environ. Microbiol., 1995, 61, 3069-3075.

30 R. F. Wang, W. W. Cao and C. E. Cerniglia, Appl. Environ. Microbiol., 1996, 62, 1242-1247.

31 X. F. Zhang, F. R. Qiu, J. Jiang, C. L. Gao and Y. Z. Tan, Xenobiotica, 2011, 41, 290-296.

32 C. Y. Lee, T. Y. Lai, M. K. Tsai, P. Ou-Yang, C. Y. Tsai, S. W. Wu, L. C. Hsu and J. S. Chen, Sci. Rep., 2016, 6, 20486.

33 F. Ashrafi, F. Kowsari, A. Darakhshandeh and P. Adibi, Int. J. Hematol. Oncol. Stem Cell Res., 2014, 8, 45-48.

34 M. L. Chen and M. S. Sundrud, Inflammatory Bowel Dis., 2016, 22, 1157-1167.

35 S. J. Ott, M. Musfeldt, D. F. Wenderoth, J. Hampe, O. Brant, U. R. Folsch, K. N. Timmis and S. Schreiber, Gut, 2004, 53, 685-693.
36 H. Iizasa, N. Genda, T. Kitano, M. Tomita, K. Nishihara, M. Hayashi, K. Nakamura, S. Kobayashi and E. Nakashima, J. Pharm. Sci., 2003, 92, 569-576.

37 A. O. Sehirli, S. Cetinel, N. Ozkan, S. Selman, S. Tetik, M. Yuksel and F. G. Dulger, J. Physiol. Pharmacol., 2015, 66, 203-214.

38 J. Kabeerdoss, V. Sankaran, S. Pugazhendhi and B. S. Ramakrishna, BMC Gastroenterol., 2013, 13, 20.

39 X. C. Morgan, T. L. Tickle, H. Sokol, D. Gevers, K. L. Devaney, D. V. Ward, J. A. Reyes, S. A. Shah, N. LeLeiko, S. B. Snapper, A. Bousvaros, J. Korzenik, B. E. Sands, R. J. Xavier and C. Huttenhower, Genome biology, 2012, 13, R79.

40 P. Gionchetti, F. Rizzello, A. Venturi and M. Campieri, J. Gastroenterol. Hepatol., 2000, 15, 489-493.

41 A. S. Neish, Gastroenterology, 2009, 136, 65-80.

42 W. Wang, L. P. Chen, R. Zhou, X. B. Wang, L. Song, S. Huang,

G. Wang and B. Xia, J. Clin. Microbiol., 2014, 52, 398-406.

43 D. Berry and W. Reinisch, Best Pract. Res., Clin. Gastroenterol., 2013, 27, 47-58.

44 H. Sokol, P. Seksik, L. Rigottier-Gois, C. Lay, P. Lepage, I. Podglajen, P. Marteau and J. Dore, Inflammatory Bowel Dis., 2006, 12, 106-111.

45 H. Sokol, B. Pigneur, L. Watterlot, O. Lakhdari, L. G. Bermudez-Humaran, J. J. Gratadoux, S. Blugeon, C. Bridonneau, J. P. Furet, G. Corthier, C. Grangette, N. Vasquez, P. Pochart, G. Trugnan, G. Thomas, H. M. Blottiere, J. Dore, P. Marteau, P. Seksik and P. Langella, Proc. Natl. Acad. Sci. U. S. A., 2008, 105, 1673116736.

46 M. Scharl and G. Rogler, Int. J. Inflammation, 2010, 2010, 671258.

47 R. Duan, N. Hu, H. Y. Liu, J. Li, H. F. Guo, C. Liu, L. Liu and X. D. Liu, Acta Pharmacol. Sin., 2012, 33, 767-774.

48 B. Bauer, A. M. Hartz and D. S. Miller, Mol. Pharmacol., 2007, 71, 667-675.

49 F. Yan, H. Cao, T. L. Cover, R. Whitehead, M. K. Washington and D. B. Polk, Gastroenterology, 2007, 132, 562-575.

50 E. F. O'Shea, P. D. Cotter, C. Stanton, R. P. Ross and C. Hill, Int. J. Food Microbiol., 2012, 152, 189-205.

51 S. P. Claus, T. M. Tsang, Y. Wang, O. Cloarec, E. Skordi, F. P. Martin, S. Rezzi, A. Ross, S. Kochhar, E. Holmes and J. K. Nicholson, Mol. Syst. Biol., 2008, 4, 219.

52 K. M. Keeney and B. B. Finlay, Curr. Opin. Microbiol., 2011, 14, 92-98.

53 B. Bahrami, S. Macfarlane and G. T. Macfarlane, J. Appl. Microbiol., 2011, 110, 353-363.

54 M. Ufer, R. Hasler, G. Jacobs, S. Haenisch, S. Lachelt, F. Faltraco, C. Sina, P. Rosenstiel, S. Nikolaus, S. Schreiber and I. Cascorbi, Pharmacogenomics, 2009, 10, 1941-1953.

55 Y. H. Li, L. Huang, X. Z. Zeng, G. P. Zhong, M. J. Ying, M. Huang and H. C. Bi, Chem.-Biol. Interact., 2014, 213, 44-50. 